\title{
Strates
}

STRATES Matériaux pour la recherche en sciences sociales

14 | 2008

Espaces du quotidien

\section{Maria Gravari-Barbas (dir.), Habiter le patrimoine : Enjeux, approches, vécu, 2005}

Aline Brochot

\section{(2) OpenEdition}

1 Journals

Édition électronique

URL : http://journals.openedition.org/strates/6713

DOI : 10.4000/strates. 6713

ISSN : $1777-5442$

Éditeur

Laboratoire Ladyss

Édition imprimée

Date de publication : 1 janvier 2008

Pagination : $277-279$

ISBN : 0768-8067

ISSN : 0768-8067

Référence électronique

Aline Brochot, « Maria Gravari-Barbas (dir.), Habiter le patrimoine : Enjeux, approches, vécu, 2005 »,

Strates [En ligne], 14 | 2008, mis en ligne le 04 mars 2013, consulté le 23 septembre 2020. URL : http:// journals.openedition.org/strates/6713; DOI : https://doi.org/10.4000/strates.6713

Ce document a été généré automatiquement le 23 septembre 2020

Tous droits réservés 


\title{
Maria Gravari-Barbas (dir.), Habiter le patrimoine : Enjeux, approches, vécu, 2005
}

\author{
Aline Brochot
}

\section{RÉFÉRENCE}

Maria Gravari-Barbas (dir.), Habiter le patrimoine : Enjeux, approches, vécu, Rennes, Presses Universitaires de Rennes, 2005, 618 p.

1 «Comment les sociétés contemporaines "habitent-elles" les lieux, les sites, les monuments qu'elles constituent en patrimoine? Comment investissent-elles le cadre matériel auquel elles attribuent une valeur patrimoniale et dans lequel elles sont, tout de même, appelées à évoluer, à circuler, à travailler, à construire, à consommer et à produire? Que peuvent nous apprendre les modalités avec lesquelles les groupes sociaux réinvestissent les lieux patrimoniaux sur les rapports que ceux-ci entretiennent avec l'espace ?». Telles étaient les questions posées par la troisième Université d'été du Val-de-Loire Patrimoine mondial d'octobre 2003 et dont l'ensemble des contributions a été rassemblé dans cet ouvrage.

2 Somme de réflexions et de points de vue à partir, le plus souvent, d'études de cas dont il faut d'emblée souligner la diversité disciplinaire et géoculturelle, les nombreuses contributions (52) ont été organisées selon quatre grands types d'approches de la question de l'habiter dans un contexte particulier, celui du « lieu patrimonial »:

\section{Sens, vécu, approches d'habiter}

3 La mise en patrimoine de son espace de vie n'est pas sans bouleverser à la fois le regard que l'on porte sur lui et la relation que l'on peut entretenir avec lui, avec l'obligation de l'inscrire dans le long terme. Avec l'attribution d'un statut patrimonial, ce sont aussi de 
nouvelles pratiques du lieu qui s'imposent aux habitants, une manière de "code d'habiter » dont il est difficile souvent de s'affranchir. C'est aussi une sorte de " moule » général dans lequel les habitants sont plus ou moins sommés de se couler, imposant des règles de vie communes qui, si elles sont créatrices d'une identité forte (le quartier du Fenêt à Saumur, la Casbah d'Alger...), placent cependant les habitants en situation de rupture par rapport à leur environnement. Bien souvent, et malgré toutes les contraintes et "pertes multiples" afférentes à ce nouvel ordre patrimonial, la dimension symbolique attachée au lieu habité permet néanmoins de transcender les partitions sociales et, individuellement et collectivement, de faire sienne la matérialité du lieu en s'appropriant son histoire. Mais « croiser l'histoire des lieux et le vécu de ceux qui les investissent [...] ne se fait pas sans heurts ni conflits » et le quotidien du lieu n'est alors qu'une série d'ajustements des pratiques actuelles non seulement à la matérialité du lieu, mais aussi à sa symbolique lorsque ce sont des idéologies de l'habiter qui ont présidé à son édification (La Cité radieuse de Le Corbusier par exemple).

4 Habiter un «lieu patrimonial ", a fortiori lorsque celui-ci est mondialement reconnu comme tel, permet aussi d'asseoir et de renforcer sa légitimité sociale, d'autant plus que le pouvoir symbolique des lieux renvoie à une histoire glorieuse (les palais royaux...). Dès lors, le partage entre le privé, le collectif et le public peut s'avérer problématique et le « lieu patrimonial » vidé d'une partie de son sens si la connexion ne peut s'établir entre ces trois ordres.

\section{Stratégies d'acteurs et enjeux politiques et sociaux}

5 Ce deuxième thème aborde la question de la place et du jeu des acteurs. Devant la multiplicité des intervenants aux intérêts multiples, voire antagonistes (élus, associations, architectes, experts internationaux, ONG...) les habitants sont parfois loin d'être les maitres du jeu. De la désignation du « lieu patrimonial » à sa gestion et à son vécu au quotidien, ce sont des rapports de force d'une grande intensité qui prennent place, car il n'y va pas seulement de la protection ou de la réhabilitation d'un patrimoine bâti, mais aussi souvent de la reproduction symbolique de formes urbaines plus ou moins idéalisées et conçues à leur origine en stricte adéquation avec leur « contenu social ». Dès lors, les rapports entre les différents acteurs et les habitants se placent inévitablement sous le signe de la tension quand ce n'est pas du conflit ouvert, chacun opposant à l'autre sa légitimité à occuper le lieu patrimonialisé et à le conformer à ses propres intérêts.

Ce qui ressort aussi avec force dans ce groupe de contributions est l'existence d'un lien quasi tautologique entre patrimonialisation et gentrification. Qu'il s'agisse du Vieux Québec, du quartier historique du Mans, d'Angers ou encore de Mexico, partout la réhabilitation et la "labellisation» patrimoniale ont été sanctionnées par un bouleversement sociodémographique qui a vu l'arrivée de populations aisées, plutôt jeunes, et la relégation progressive des plus démunis incapables de suivre le nouveau "train de vie» de leur quartier impulsé par ces nouveaux habitants. La pression immobilière est telle que les élus, partagés entre la volonté de requalifier les centresvilles et celle d'assurer une certaine mixité sociale, ne disposent que d'une marge de manœuvre extrêmement limitée. 


\section{Habiter c'est aussi recevoir... le rapport à l'altérité}

7 Il est désormais commun de dire que c'est le regard extérieur qui construit l'identité et la valeur d'un lieu, bien plus que les pratiques et représentations de ses habitants. Les contributions regroupées dans ce thème ne vont pas à l'encontre de cette affirmation. $\mathrm{Si}$, dans un premier temps, les touristes ou étrangers à la recherche d'un lieu de villégiature et les immigrés de retour ont contribué à forger ce regard et ce jugement de valeur en investissant des «lieux délaissés» par les habitants eux-mêmes, les institutions internationales (Unesco) ont à leur tour largement contribué à amplifier le processus de désignation-révélation du « lieu patrimonial » en y ajoutant l'impératif du partage et une certaine obligation de mise à disposition pour un public extérieur.

8 Cette reconnaissance par l'Autre n'est pas sans générer des discours et des comportements chargés d'ambiguïté : Entre sentiment de dépossession et perspective de plus-value à tirer de l'exploitation de la rente de situation, le jeu et le discours des acteurs ne sont bien entendu pas neutres; il s'agit d'organiser la rencontre - à défaut de pouvoir l'éluder -, de la rendre féconde quitte, pour certains, à suivre des «stratégies économiques qui ne s'embarrassent ni des habitants ni de la réalité architecturale ».

9 Alors quid de cette rencontre lorsqu'elle prend la forme d'une intrusion qui menace l'intégrité physique et sociale du lieu? Lorsque le différentiel socioculturel est tel qu'elle doive passer par un jeu de rôles stéréotypé sans réelle imprégnation ou échange possibles entre les « accueillants » et les « accueillis »?

\section{« Habiter » un lieu de production et de travail}

Comment convertir un espace de production industrielle en lieu patrimonial? Comment faire de lieux généralement voués à la démolition des lieux de vie qui aient $\mathrm{du}$ sens et un avenir? Telles sont les questions posées dans ce dernier groupe de contributions.

11 D'emblée, l'idée que pour pouvoir être patrimonialisés, les sites industriels doivent avoir perdu leur fonction semble s'imposer. Pourtant, cette patrimonialisation ne peut se faire sans un projet qui fasse appel à la fois à la mémoire du lieu et à sa symbolique. Il $\mathrm{y}$ a, comme dans le cas des bassins miniers et sidérurgiques, des enjeux forts à " conserver des emprises qui ont structuré la société et marqué les paysages » et à les constituer en ressource. Plus que sur leur mémoire vécue, il semble que ce soit davantage sur la matérialité des sites eux-mêmes dans leur architecture et dans leur génie technique que repose la légitimation de cette construction de la ressource patrimoniale, au moins dans un premier temps. Les porteurs initiaux du projet sont ensuite relayés par les pouvoirs publics ou les entrepreneurs privés qui, dès lors que la potentialité de la ressource est avérée, peuvent s'engager dans une démarche de revalorisation/réinvestissement du site et dans la construction d'une image qui va pouvoir, in fine, servir une logique marchande.

Deux modes de réappropriation et de recyclage des sites industriels sont bien mis en évidence dans les cas présentés ici. Le premier s'appuie sur la mémoire ouvrière présentée comme allégorique de cohésion sociale, d'esprit communautaire et d'ancrage dans le lieu; lien que les entreprises muséographiques s'attachent le plus souvent à 
restituer et à préserver. Le deuxième mode s'appuie au contraire sur un changement radical d'affectation du site. Dépouillé de son contenu matériel qui permettait d'en décrypter le sens, et ainsi libéré d'un "devoir de mémoire " parfois difficile à assumer, le site industriel devient un espace vierge voué à la création artistique, considérée comme une manière de transfiguration du lieu. Le recyclage de locaux ou sites industriels, qu'il soit muséographique ou artistique, permet alors la neutralisation de la mémoire et éloigne le risque de voir ressurgir « la violence des rapports sociaux » qui lui est attachée.

13 L'intérêt de cet ouvrage tient d'abord à la très grande diversité des cas de figure présentés qui, chacun dans son contexte propre, contribue à faire comprendre ce qui se joue au quotidien entre des individus, des groupes sociaux ou toute une société locale dans leurs rapports avec leur espace de vie lorsque celui-ci se voit doté d'une valeur patrimoniale. De l'indifférence à la réappropriation, de l'acceptation à l'opposition, du réinvestissement au changement d'affectation, d'ajustements ponctuels à la mise en place de réglementations contraignantes instruites sur le long terme, "habiter le patrimoine » ne va pas de soi, nulle part. Car l'autre intérêt majeur de ces contributions est de montrer que, quels que soit l'échelle, la situation géographique, le contexte social, culturel et politique, c'est une très grande similitude de réponses qui sont apportées par les habitants à la patrimonialisation de leurs lieux de vie.

L'une des grandes lignes de fond qui traverse l'ensemble de ces contributions est la mise en évidence de ce que nous pourrions appeler les «effets pervers de la patrimonialisation » qui, souvent loin de pacifier les relations entre les groupes sociaux aurait plutôt tendance à générer ou réactiver les antagonismes entre porteurs d'intérêts divergents. Et, Maria Gravari-Barbas le souligne en conclusion, c'est le plus souvent sur le mode du conflit que la patrimonialisation s'opère. Les pratiques mises en place autour du patrimoine afin d'assurer sa préservation et sa gestion, en modifiant les équilibres en place, génèrent des tensions qui peuvent aller de l'affrontement entre groupes (habitants/élus, autochtones/étrangers ", habitants/touristes) jusqu'à à l'éviction d'une partie des habitants, souvent les plus démunis. Qu'apporte alors la patrimonialisation d'un lieu si, pour certains, elle le rend « inhabitable » au quotidien ? Le coût social de la patrimonialisation apparaît donc encore à mesurer, autrement que par les entrées plus convenues de la valorisation économique et commerciale et du développement culturel et local.

Une dernière remarque s'impose une fois cet ouvrage refermé : Dans leur très grande majorité, les articles rassemblés ici renvoient essentiellement au patrimoine bâti, architectural et urbain. Faut-il y voir une certaine inertie dans les représentations qui, malgré l'élargissement considérable $d u$ champ $d u$ patrimoine, fait encore spontanément associer le patrimoine à l'urbain, à travers ses monuments ? La notion d'habiter ne peut-elle se concevoir autrement qu'en termes d'habitation? Voilà en tout cas qui ne peut manquer d'interpeller à l'heure où l'Unesco entend faire une plus grande place aux "paysages culturels " - dont beaucoup sont agricoles - et où la Convention européenne du Paysage réaffirme que le "paysage [...] représente une composante fondamentale du patrimoine culturel et naturel de l'Europe ». Il y aurait sans doute là de nouvelles pistes à explorer. 


\section{AUTEURS}

\section{ALINE BROCHOT}

Laboratoire Ladyss

CNRS, Université de Paris 1

2 rue Valette,

75005 - Paris

abrochot@univ-paris1.fr 